Research Article

\title{
Generation-Load Coordinative Scheduling considering the Demand-Response Uncertainty of Inverter Air Conditioners
}

\author{
Wei Hu, ${ }^{1}$ Jin Yang, ${ }^{2}$ Yi Wu, ${ }^{1}$ Weiguo Zhang, ${ }^{3}$ Xueming Li, ${ }^{3}$ Xiaorong $\mathrm{Li},{ }^{1}$ and Ciwei Gao ${ }^{4}$ \\ ${ }^{1}$ Power Dispatch Center, State Grid Jiangsu Electric Power Company, Nanjing 211106, China \\ ${ }^{2}$ Jiangsu Xingli Construction Group Company, Nanjing 210000, China \\ ${ }^{3}$ Power Consumption Department, Nari Technology Company, Nanjing 211106, China \\ ${ }^{4}$ School of Electrical Engineering, Southeast University, Nanjing 210096, China \\ Correspondence should be addressed to Ciwei Gao; ciwei.gao@seu.edu.cn
}

Received 31 May 2020; Accepted 22 June 2020; Published 26 July 2020

Guest Editor: Sanghyuk Lee

Copyright $\odot 2020$ Wei Hu et al. This is an open access article distributed under the Creative Commons Attribution License, which permits unrestricted use, distribution, and reproduction in any medium, provided the original work is properly cited.

\begin{abstract}
Inverter air conditioners (IACs) have gradually become the mainstream of resident air-conditioning equipment. Similar to traditional fixed-frequency air conditioners, IACs have the potential for demand response and load scheduling. However, the uncertainty of IACs is nonnegligible in generation-load scheduling. In this paper, the uncertain demand-response cost of IACs is studied for the first time. Meanwhile, based on the cost, a generation-load coordinative day-ahead scheduling model is proposed. In the scheduling, an IACs aggregator and traditional generators are coordinately dispatched to minimize the expected scheduling cost of the power system. The case study shows that the coordinative scheduling model can reduce the scheduling cost of the power system and encourage the IACs aggregator to improve their responsiveness or reduce their uncertainty, so as to improve the economy and reliability of power scheduling.
\end{abstract}

\section{Introduction}

Increasing renewable energy generation $[1,2]$ and load [3] have led to difficulty in power dispatch, maintenance, and safe operation. To address the problem, more reserve capacity and generating capacity are invested, however, which lack reliability in the near term and economy in the long term [4]. In recent years, air-conditioning load is gradually increasing, and it becomes a considerable component of the maximum load. Particularly, in the summer of East China, the proportion of air-conditioning load in the maximum load can reach 50\% [5]. From the perspective of generation and load scheduling, air-conditioning load can adjust demand and provide demand response to schedulers with appropriate control strategies [6, 7]. For example, air-conditioning load can cut peak and fill valley [8], provide system backup [9], and stabilize the fluctuation of renewable energy output [10]. In summary, it is a kind of cost-effective resources suitable for system operators scheduling.

In recent years, load scheduling is gradually incorporated into the day-ahead economic dispatch of power system.
A residential load-scheduling approach is proposed to offset the uncertainty caused by renewable generation in [11]. Sohet et al. [12] design an incentive mechanism to foster engagement of electric vehicles in demand response and load scheduling. An optimal day-ahead scheduling of power-to-gas (PtG) storage and gas load management is proposed in [13], where PtG is regarded as a schedulable resource. In literature, load-scheduling optimization is used for the economic evaluation of demand-response load. Since air conditioners have the great potential to offer demand-response service, some studies have proposed the methodology of scheduling optimization for them [14-16]. However, inverter air conditioners are rarely incorporated into the day-ahead scheduling of power system as a kind of schedulable load or response-demand resource, where the control strategy, controllable duration, and uncertainty should be all considered in it.

Dynamic performance, stability, sensitivity, and robustness are intensively studied in [17]; IACs are proved to be suitable for providing regulation capacity to power systems. A priceresponsive model-based optimal demand-response control strategy is proposed in [18] for IACs to reduce electricity costs 
and the peak power demands. Although the modeling and control strategies of IACs are intensively studied in the literature, the uncertainty in demand response of IACs is ignored, which is harmful to the stability of power system or causes more expensive cost in purchasing reserve.

Meanwhile, the modeling, control strategies, and reserve optimization of inverter air conditioners are studies for demand response in $[19,20]$ and for frequency regulation in [21-23]. Although, in the literature, the feasibility and technologies of inverter air conditioners to provide demand-response service have been fully studied, the economic evaluation and load-scheduling methodology are still lack of consideration, especially when the uncertainty is regarded as a kind of risk cost for the scheduler. Hence, the uncertain-risk cost should be modeled and incorporated into the scheduling optimization of inverter air conditioners for power system.

To address the above problems, this paper proposes a risk evaluation-based generation-load collaborative scheduling optimization model, where the objective function is to minimize the scheduling cost of power system and the solution is subject to the operational reliability of power system. The contributions are as follows:

(1) From the perspective of the scheduler, this paper proposes a payment cost model of IACs considering the uncertainty in demand response for the first time, which includes the payment cost of demand response and uncertain-risk cost. It can be applied to evaluate the scheduling cost of IACs with the scheduling potential of different degrees. This provides methodology of economic evaluation for generation-load coordinative scheduling.

(2) Based on the payment cost model of IACs, a generation-load coordinative scheduling optimization is also formulated, where the operational reliability, controllable duration, and actual response are all taken into account, so as to efficiently evaluate the scheduling cost of power system and keep as close as possible to optimal solution.

\section{Load-Scheduling Modeling of IACs Aggregator considering Demand- Response Uncertainty}

2.1. Stochastic Modeling of IAC. When an IAC is turned on, the relationship between the temperature of the room and the cooling capacity is given in

$$
\begin{aligned}
T_{\text {in }}[(n+1) \Delta t]= & e^{-\Delta t / \mathrm{RC}} T_{\text {in }}(n \Delta t) \\
& +\left(1-e^{-\Delta t / \mathrm{RC}}\right)\left[T_{\text {out }}(n \Delta t)-Q_{\mathrm{AC}}(n \Delta t) R\right] \\
& +V(n \Delta t),
\end{aligned}
$$

where $T_{\text {in }}(n \Delta t)$ and $T_{\text {out }}(n \Delta t)$ denote the indoor and outdoor temperature $\left({ }^{\circ} \mathrm{C}\right)$ at $n \Delta t ; Q(n \Delta t)$ denotes the cooling capacity $(\mathrm{kW})$ of the IAC at $n \Delta t ; \Delta t$ denotes the temperature-monitoring interval $(s)$ of the IAC; $R$ is the equivalent thermal resistance $(\Omega)$ of the room; C is the equivalent heat capacity $\left(\mathrm{J} /{ }^{\circ} \mathrm{C}\right)$ of the room; $V(n \Delta t)$ denotes the random temperature deviation caused by the influence of outdoor airflow movement/sunlight exposure, window opening and closing, indoor personnel flow, and other factors.

During the operation of IACs, the cold/hot air intake is controlled by adjusting the operating frequency of compressor, so as to continuously control the power. The relationship between the operating frequency, cooling capacity, and electric power of an IAC is shown in

$$
\begin{aligned}
& Q_{\mathrm{AC}}(n \Delta t)=\alpha f(n \Delta t)+\beta, \\
& P_{\mathrm{AC}}(n \Delta t)=\gamma f(n \Delta t)+\delta,
\end{aligned}
$$

where $P_{\mathrm{AC}}(n \Delta t)$ denotes the cooling power $(\mathrm{kW})$ of the IAC at $n \Delta t ; f(n \Delta t)$ denotes the operating frequency of the compressor $(\mathrm{Hz})$ at $n \Delta t ; \alpha$ and $\beta$ denote the constant coefficient of the cooling capacity; $\gamma$ and $\delta$ denote the constant coefficient of the active power.

2.2. Modeling of Demand-Response Uncertainty for IACs Aggregator. In this paper, we adopt a statistical methodology to evaluate expected uncertain-risk cost of IACs-load scheduling. Also, the confidence level is considered in riskrelated constraints. To apply the methodology to IACs-load scheduling, we consider stochastic processes in two aspects: one is the responsive deviation of signal IAC; the other is the responsive deviation of IACs aggregator. The detailed methodology and application are as follows: we derive demand-response potential (the expected responsive power) and risk (the distribution and variance of responsive power) of single IAC from the modeling as presented in formulas (1)-(9); then the stochastic uncertain-risk cost of IACs aggregators is deduced as presented in formulas (10)-(14).

Given that the outdoor temperature $T_{\text {out }}$ is unchanged during the minimum scheduling period and the IAC operates under the setting temperature $T_{\text {set }}$ before load scheduling, the load power $P_{\mathrm{AC}}$ can be formulated, as expressed in formula (4); when the scheduling instruction is issued, taking load reduction as an example, the IAC will operate under the lowest frequency $f_{\text {min }}$; the load power $P_{\text {AC }}$ can be formulated, as expressed in formula (5); when the indoor temperature gradually rises to the upper bound $T_{\max }$ beyond the range of comfortable temperature for users, the scheduler will release the control and readjust the frequency of the IAC to the $f_{\text {set }}$ corresponding to $T_{\text {set }}$. The adjustment process is presented in Figure 1:

$$
\begin{aligned}
P_{\text {AC }} & \left(T_{\text {set }}, T_{\text {out }}, V(n \Delta t)\right) \\
& =\gamma\left(\frac{T_{\text {out }}-T_{\text {set }}+\left(V(n \Delta t) /\left(1-e^{-\Delta t / \mathrm{RC}}\right)\right)}{\alpha R}-\frac{\beta}{\alpha}\right)+\delta,
\end{aligned}
$$

$P_{\text {AC }}\left(f_{\text {min }}\right)=\gamma \cdot f_{\text {min }}+\delta$.

Under the control mode, the controllable duration $t_{c}$ of an IAC can be expressed in 


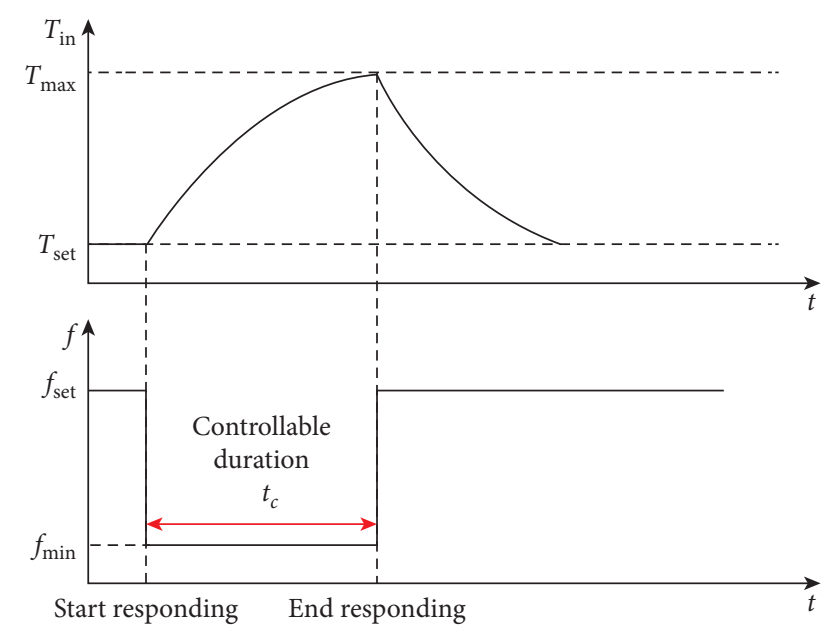

Figure 1: The load-scheduling process of a IAC.

$$
t_{c}=\mathrm{RC} \cdot \ln \frac{T_{\text {out }}-T_{\text {set }}-R Q_{\min }+V(n \Delta t)}{T_{\text {out }}-T_{\max }-R Q_{\min }} .
$$

The load reduction or reserve capacity of an IAC can be expressed in

$$
\begin{aligned}
\Delta P_{\mathrm{AC}} & =P_{\mathrm{AC}}\left(T_{\text {set }}, T_{\text {out }}, V(n \Delta t)\right)-P_{\mathrm{AC}}\left(f_{\text {min }}\right) \\
& =\gamma \cdot\left(\frac{T_{\text {out }}-T_{\text {set }}}{\alpha R}-\frac{\beta}{\alpha}\right)-\gamma \cdot f_{\text {min }}+\frac{\gamma \cdot V(n \Delta t)}{\alpha R\left(1-e^{-\Delta t / R C}\right)} .
\end{aligned}
$$

$\gamma \cdot V(n \Delta t) / \alpha R\left(1-e^{-\Delta t / R C}\right)$ is a stochastic function, denoting responsive deviation of the IAC, denoted as $\xi(n \Delta t)$ :

$$
\xi(n \Delta t)=\frac{\gamma}{\alpha R\left(1-e^{-\Delta t / R C}\right)} V(n \Delta t)
$$

In this paper, the normal distribution is used to evaluate the stochastic responsive deviation of a single IAC.

For an aggregator with $N$ IACs, the mean value $\xi_{i}$ of each IAC is 0 , and the variance of each IAC is denoted by $\sigma_{\xi, i}(i=1,2, \cdots, N)$. Then, the mean responsive deviation of the aggregator is also 0 ; the variance is expressed by

$$
\sigma=\sqrt{\sum_{i=1}^{N} \sigma_{\xi, i}^{2}}
$$

Here, the demand-response-implementation mechanism is introduced as follows. Before participating in load scheduling, an IACs aggregator will contract with IAC users to control over them during load-peak periods. Only during the periods, when the aggregators receive demand-response (DR) signals, they can control the power of the IACs. After $\mathrm{DR}$, the aggregators should pay to the users or give a discount on electricity fee according to the contracts. Moreover, to evaluate the DR potential of IACs, IACs should update and report their operational status to the aggregator every scheduling period.

\section{Stochastic Cost of IACs Aggregator for Load Scheduling}

3.1. Payment Cost. In the traditional load-scheduling contract between the system operator and users, after the airconditioning load responds to the scheduling instruction, the system operator needs to pay a certain fee to compensate the load according to the actual response of the load. The responsive deviation of IACs aggregator will affect the final clearing expenses; hence, the uncertainty of the payment cost should be also considered by the scheduler. The payment cost $C_{\text {pay }}$ of $l$ th IACs aggregator at $t$ th scheduling period consists of two parts: one is compensation fee paid to users according to the actual response; the other is the penalty fee caused by the responsive uncertainty of the aggregator, which is revenue for schedulers, as expressed in

$$
C_{\text {pay }}(l, t)=P_{\text {real }}(l, t) \cdot v_{c}(l, t)+\xi_{l} \cdot v_{p}(l, t),
$$

where $v_{c}$ and $v_{p}$ denote the capacity price and penalty price for IACs aggregator, respectively; $P_{\text {real }}$ denotes the actual responsive power of the IACs aggregator, which is the difference between expected load reduction $P_{\text {de }}$ and responsive deviation, as expressed in formula (11). $\xi_{l}$ denotes the mean responsive deviation of $l$ th IACs aggregator:

$$
P_{\text {real }}(l, t)=P_{\text {de }}(l, t)+\xi_{l} \text {. }
$$

Take (11) into (10) and derive the following formula:

$$
C_{\text {pay }}(l, t)=P_{\mathrm{de}}(l, t) \cdot v_{c}(l, t)+\xi_{l} \cdot\left[v_{c}(l, t)+v_{p}(l, t)\right] \text {, }
$$

where $P_{\mathrm{de}}(l, t) \cdot v_{c}(l, t)$ is denoted by $C_{\mathrm{fix}}$ and $\xi_{l} \cdot\left[v_{c}(l, t)+\right.$ $\left.v_{p}(l, t)\right]$ is denoted by $C_{\xi}$. Then, the expected payment cost $C_{\text {pay }}$ of $l$ th IACs aggregator at $t$ th scheduling period is expressed by

$$
E\left[C_{\text {pay }}(l, t)\right]=C_{\text {fix }}(l, t)+E\left[C_{\xi}(l, t)\right]
$$

where $E\left(C_{\xi}\right)$ can be expressed by

$$
\begin{aligned}
E\left[C_{\xi}(l, t)\right]= & \int_{-\infty}^{-P_{\mathrm{de}}(l, t)}\left[\xi_{l} \cdot v_{p}(l, t)-P_{\mathrm{de}}(l, t) \cdot v_{c}(l, t)\right] \cdot f\left(\xi_{l}\right) d \xi_{l} \\
& +\int_{-P_{\mathrm{de}}(l, t)}^{0} \xi_{l} \cdot\left[v_{c}(l, t)+v_{p}(l, t)\right] \cdot f\left(\xi_{l}\right) d \xi_{l},
\end{aligned}
$$

where $f\left(\xi_{l}\right)$ denotes the probability density function of responsive deviation of $l$ th IACs aggregator.

3.2. Uncertain-Risk Cost. There is always a deviation between the actual response and the expected response of IACs aggregators, which brings a certain risk and corresponding cost to the scheduler. When the responsive power is less than the expected responsive power, the system may not be able to supply enough power to users, resulting in power failure. To supply enough power and maintain supply-demand balance, schedulers need to bear the payment risk of direct load control (DLC). Hence, power-failure loss can be evaluated by the compensation cost of DLC; when the aggregator 
overresponds, the power supply is greater than the demand, and the power grid needs to bear the loss of reduced electricity sales. In summary, for a scheduler, the uncertain-risk cost $C_{\text {risk }}$ caused by the responsive uncertainty of $l$ th IACs aggregator at $t$ th scheduling period can be expressed by

$$
\begin{aligned}
C_{\text {risk }}(l, t)= & \int_{-\infty}^{0}-\xi_{l} \cdot v_{\text {re }}(l, t) \cdot f\left(\xi_{l}\right) d \xi_{l} \\
& +\int_{0}^{+\infty} \xi_{l} \cdot v_{\text {sell }}(l, t) \cdot f\left(\xi_{l}\right) d \xi_{l},
\end{aligned}
$$

where $v_{\text {re }}$ denotes the power-failure loss per capacity $(\mathrm{RMB} / \mathrm{kWh}) ; v_{\text {sell }}$ denotes the loss of reduced electricity sales per capacity (RMB/kWh).

\section{Generation-Load Coordinated Scheduling Optimization}

Figure 2 illustrates the generation-load coordinated scheduling steps in this paper. First of all, before day-ahead scheduling, IACs aggregators should submit demandresponse parameters to scheduler, such as bidding price $v_{c}(l, t)$ (capacity price), mean responsive deviation $\xi_{l}$, and the probability density function of responsive deviation $f\left(\xi_{l}\right)$, which are derived according to formulas (1)-(9). At the same time, traditional generators also submit generating parameters to the scheduler. Secondly, the optimal scheduling plan will be calculated according to formulas (16)-(27) and then be issued to the generators and IACs aggregators. Finally, the generators and IACs aggregators will execute the scheduling plan. Finally, scheduler will conduct settlement according to actual generating capacity of generators and responsive deviation of IACs aggregators.

4.1. Objective Function. The objective function of the generation-load coordinated scheduling optimization is to minimize the expected generation cost and load-scheduling cost:

$$
F=\min \sum_{t=1}^{T} \sum_{j=1}^{N_{G}} C_{G_{j}, t}\left(P_{G}(j, t)\right)+E \sum_{t=1}^{T} \sum_{l=1}^{N_{L}}\left[C_{\text {total }}(l, t)\right],
$$

where $T$ is the number of scheduling periods; $N_{G}$ and $N_{L}$ denote the number of generators and IACs aggregators, respectively; $C_{G j}, t\left(P_{G}(j, t)\right)$ is the generationcost function of $j$ th generator at $t$ th period, which is presented in

$$
C_{G j, t}\left(P_{G}(j, t)\right)=a_{j} P_{G}(j, t)^{2}+b_{j} P_{G}(j, t)+c_{j} s_{G}(j, t),
$$

where $P_{G}(j, t)$ denotes the power of $j$ th generator at $t$ th period; $S_{G}(j, t)$ denotes the operational status of $j$ th generator at $t$ th period, which is equal to 0 when it is off and 1 when it is on; $a_{j}, b_{j}$, and $c_{j}$ denote the parameters of the generation-cost function of $j$ th generator.
The scheduling cost $C_{\text {total }}$ of IACs aggregators consists of the payment cost and the uncertain-risk cost, as presented in

$$
C_{\text {total }}(l, t)=C_{\text {fix }}(l, t)+C_{\xi}(l, t)+C_{\text {risk }}(l, t) .
$$

Above all, the objective function can be simplified as expressed by

$$
\begin{aligned}
F= & \min \sum_{t=1}^{T} \sum_{j=1}^{N_{G}} C_{G j, t}\left(P_{G}(j, t)\right)+\sum_{t=1}^{T} \sum_{l=1}^{N_{L}} C_{\text {fix }}(l, t) \\
& +E\left[C_{\xi}(l, t)\right]+C_{\text {risk }}(l, t) .
\end{aligned}
$$

The risk factor $\lambda$ is introduced into formula (19), denoting the sensitivity of the scheduler to the risk. The objective function can be expressed as follows:

$$
\begin{aligned}
F= & \min \sum_{t=1}^{T} \sum_{j=1}^{N_{G}} C_{G j, t}\left(P_{G}(j, t)\right)+\sum_{t=1}^{T} \sum_{l=1}^{N_{L}}(1-\lambda) C_{\text {fix }}(l, t) \\
& +\lambda\left\{E\left[C_{\xi}(l, t)\right]+C_{\text {risk }}(l, t)\right\} .
\end{aligned}
$$

\subsection{Constraints}

4.2.1. Power-Balance Constraint. Generation should be equal to consumption at each scheduling period:

$$
\sum_{j=1}^{N_{G}} P_{G}(j, t)+\sum_{l=1}^{N_{L}} P_{\mathrm{de}}(l, t)=P_{D}(t)
$$

where $P_{D}(t)$ denotes forecasted load at $t^{\text {th }}$ period.

\subsubsection{Constraints of Generators}

(1) Generation capacity constraint is as follows:

$$
P_{G j, \min } \leq P_{G}(j, t) \leq P_{G j, \max }
$$

where $P_{G J \text {, min }}$ and $P_{G J \text {, max }}$ denote the upper and lower bound of $j$ th generating power, respectively.

(2) Ramping constraint is as follows:

$$
-D_{R j} \leq P_{G}(j, t)-P_{G}(j, t-1) \leq U_{R j},
$$

where $U_{R j}$ and $D_{R j}$ denote the maximum ramp-up and ramp-down rate of $j$ th generator, respectively.

\subsubsection{Constraints of IACs Aggregator}

(1) Responsive power constraint is as follows:

$$
0 \leq P_{\text {de }}(l, t) \leq P_{l, \text { max }},
$$

where $P_{l, \max }$ denotes the maximum responsive power of $l$ th aggregator.

(2) Controllable duration constraint is as follows: 


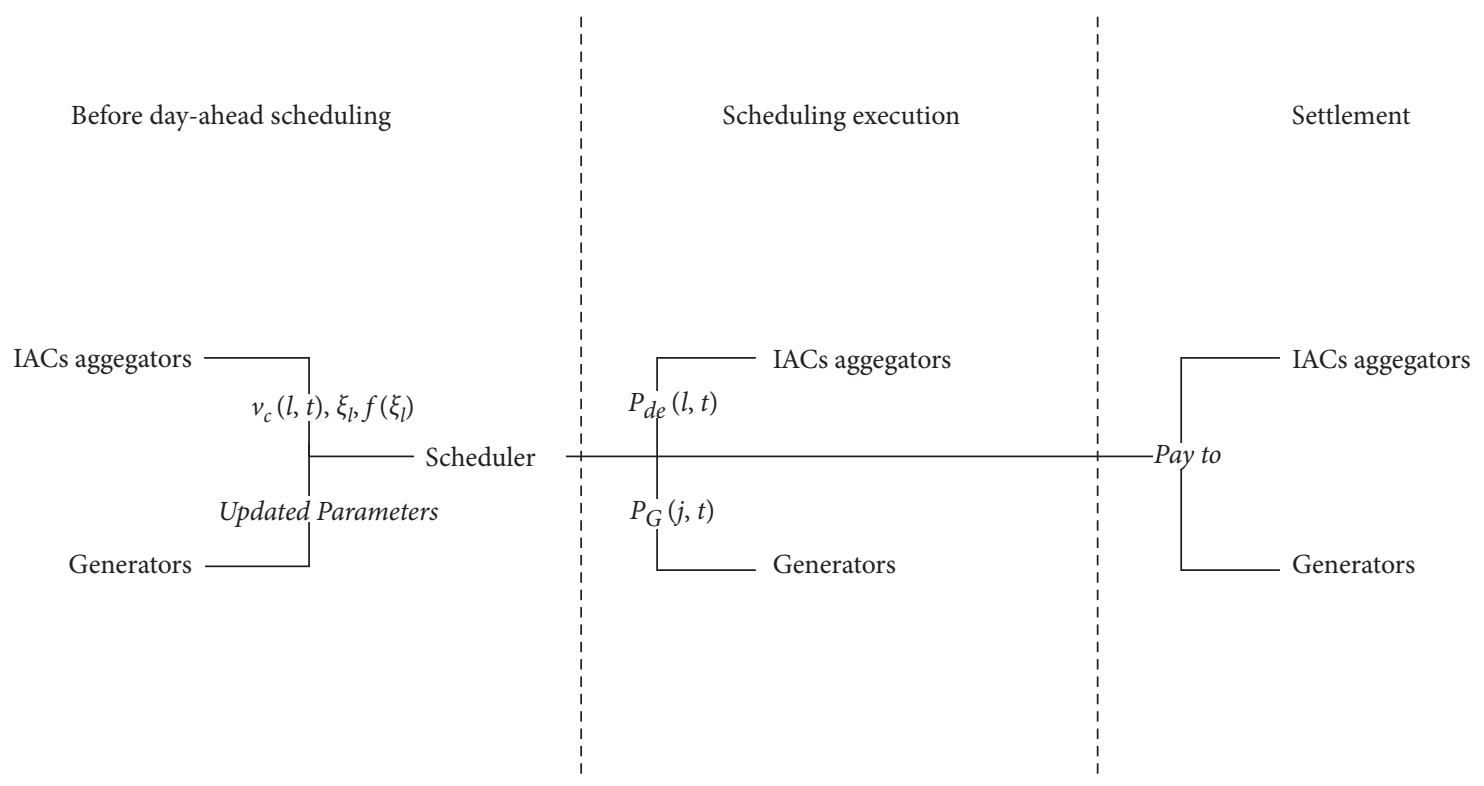

Figure 2: Coordinated scheduling steps.

$$
t_{\text {control }}(l, t) \geq \Delta T
$$

where $t_{\text {control }}(l, t)$ denotes the controllable duration of $l$ th aggregator during $t$ th scheduling period; $\Delta T$ denotes the scheduling interval, which is $30 \mathrm{~min}$ in this paper.

(3) Responsive stability constraint is as follows:

To ensure the stability of system operation, chance constraint method is adopted to constrain the responsive stability of IACs aggregators. Namely, the probability that the responsive-deviation rate of IACs aggregator is less than or equal to a set value $\varepsilon^{*}$ is not less than $\beta$ :

$$
P_{r}\left[\varepsilon_{l} \leq \varepsilon^{*}\right] \geq \beta,
$$

where $\varepsilon_{l}$ denotes the responsive-deviation rate of $l$ th aggregator, which is the ratio of the variance of the responsive-deviation power to expected load reduction:

$$
\varepsilon_{l}=\frac{\sigma\left(\xi_{l}\right)}{P_{\mathrm{de}}(l, t)}
$$

Formulas (19)-(27) constitute a generation-load coordinated scheduling optimization model considering the uncertainty of air-conditioning response. The decision variables include the output of the traditional generating units $P_{G}(j, t)$ and the expected load reduction of IACs aggregators $P_{\text {de }}(j, t)$. The model is a nonlinear stochastic optimization based on chance constraints.

\section{Case Study}

5.1. Numerical Setting. Taking IACs aggregators participating in system scheduling in a typical high-temperature day of summer as a scenario, the scheduler forecasts the load power of the next day in the region and obtains the daily load prediction curve as shown in Figure 3. It is assumed that there are 3 traditional generating units in this area; see Table 1 for details. The sum of the maximum output of the three units is $2000 \mathrm{~kW}$, which cannot meet the power supply during the peak-load period. There will be a power supply gap between 12:00 and 15:00. In the period of power supply shortage, the traditional generating units and IACs aggregators are jointly scheduled to meet the load requirements.

According to the set temperature value and actual responsive characteristics, the IACs are aggregated into three aggregators, and the specific information of each aggregator is shown in Table 2.

5.2. Potential of Load Reduction. According to the weather forecast, the outdoor temperature of the next day when there is a power supply gap in the area is obtained, as shown in Figure 4. Based on the responsive uncertainty model of IACs aggregator, the load-reduction potential, controllable duration, and the scheduling responsive-deviation distribution of IACs aggregators are calculated, as shown in Table 3.

It can be observed from Table 3 that, in each period of power supply gap, the controllable durations of aggregators $\mathrm{A}, \mathrm{B}$, and $\mathrm{C}$ are more than 30 minutes, which can cover the duration of each scheduling interval and can meet the comfort experience of IAC users in the process of scheduling.

5.3. Results and Analysis. Based on the generation-load coordinated scheduling optimization model considering the uncertainty of IACs, the output of the traditional generators at each time is optimized, and the results are shown in Figure 5. The overall coordinated scheduling results of IACs aggregators and traditional units are shown in Figure 6.

It can be seen from Figure 6 that the output of traditional generating units is different from each other at all periods, 


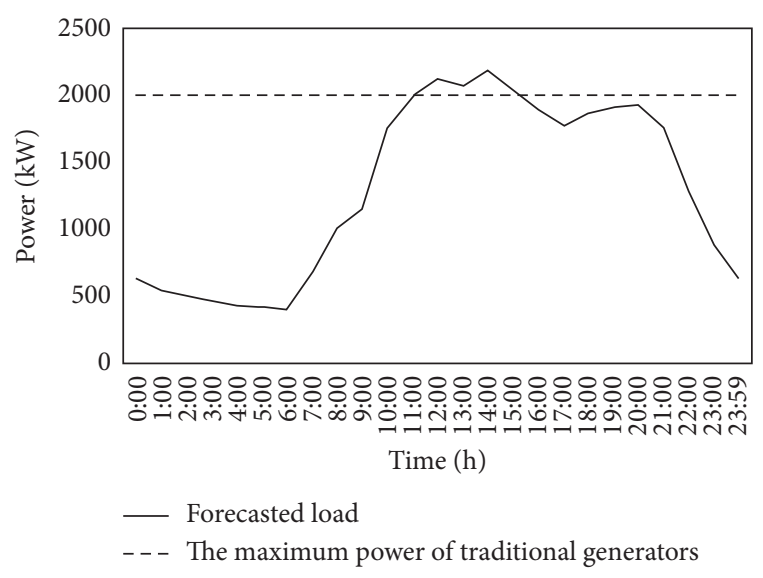

FIgure 3: Daily load prediction curve.

TABLE 1: Temperature and wildlife count in the three areas covered by the study.

\begin{tabular}{lcccc}
\hline Index & \multicolumn{3}{c}{ Parameters of generation-cost } & Maximum \\
& \multicolumn{4}{c}{$\begin{array}{c}\text { function } \\
\text { power }(\mathrm{kW})\end{array}$} \\
\hline 1 & $a_{j}$ & $b_{j}$ & $c_{j}$ & \\
2 & 0.0015 & 0.03 & 30 & 1000 \\
3 & 0.0021 & 0.02 & 60 & 550 \\
\hline
\end{tabular}

but the relationship between the output of three units is the same at each period (The Output of Generator $1>$ The Output of Generator $2>$ The Output of Generator 3), which is related to the cost coefficients of each unit. The smaller the cost coefficient, the more the output.

Most of the time, the traditional generating units can meet the load demand of the region. In the peak-load period, the sum of the maximum output of the traditional generating units is less than the forecasted load. In Figure 6, the red shaded part is the power supply gap in the peak-load period. In the power supply gap period, traditional units and aggregators need to be jointly scheduled to ensure the power supply and demand balance. See Table 4 for the total expected load reduction of aggregators in each power supply gap period.

From Table 4, the total load-reduction potential of three aggregators in each power supply gap period is greater than the power supply gap, which provides the possibility for IACs aggregators to participate in grid coordinated scheduling.

5.4. Comparison of Optimal Scheduling under Different Objectives. Two cases with different objective functions are given as follows.

Case 1. To minimize the payment cost Ctotal of IACs aggregators.

Case 2. To minimize the payment cost Cfix of IACs aggregators.
The simulation results, namely, the optimal load-reduction distributions of aggregators under different objectives, are shown in Figure 7. See Table 5 for the cost of aggregators under different objectives.

It can be seen from Figure 7 that, in Case 2, the scheduling sequence of aggregators is arranged according to the user compensation cost at each period, and aggregator with the lowest user compensation cost is called first, while, in Case 1, the user compensation cost and responsive deviation of each aggregator are taken into account for optimizing scheduling. Namely, the smaller the responsive deviation is, the more likely the aggregator will be called.

In Case 2, since the scheduler only considers the user compensation cost of aggregators, the compensation cost (1018 RMB) under the scheduling optimization is lower than the cost in Case 1. It can also be seen from Figure 7 that, in Case 2, the scheduler preferentially calls the aggregator with lower compensation cost. However, the aggregators have higher uncertain-risk cost, which causes the scheduler to bear higher payment cost (1275.7 RMB). Although the compensation cost (1031 RMB) of the aggregators in Case 1 is higher, the scheduler calls the aggregators with less responsive deviation, and the lower uncertain-risk cost makes the payment cost (1132.4 RMB) borne by the scheduler lower than that in Case 2, which proves that generation-load coordinated scheduling optimization model proposed considering the uncertainty of demand response can reflect the scheduling cost of IACs aggregators more reasonably.

\subsection{Sensitivity Analysis}

5.5.1. Payment Prices. The payment prices of schedulable load in different regions are different from each other. We assume that the payment prices of aggregators $\mathrm{B}$ and $\mathrm{C}$ are fixed, which are $3 \mathrm{RMB} / \mathrm{kWh}$ and $4 \mathrm{RMB} / \mathrm{kWh}$, respectively. The payment price of aggregator $\mathrm{A}$ ranges from 1.2 to 3.2 $\mathrm{RMB} / \mathrm{kWh}$. The simulation results show the total scheduling cost and payment cost of aggregator $\mathrm{A}$, as presented in Figure 8 .

It can be observed from Figure 8 that the payment cost and payment cost of aggregator $A$ increase with the increase of user payment price. This is because the payment price of aggregator $\mathrm{A}$ is lower than that of aggregator $\mathrm{C}$, while its responsive deviation is between $\mathrm{B}$ and $\mathrm{C}$; hence, its scheduling priority is still lower than that of aggregator $\mathrm{B}$. With the increase of user payment price, the load reduction of aggregator A remains unchanged, the expected uncertain-risk cost remains unchanged, and the fixed payment cost increases, so its payment cost and payment cost increase accordingly.

5.5.2. Penalty Prices. Similarly, suppose that the penalty price of aggregators $\mathrm{B}$ and $\mathrm{C}$ is fixed, which are $15 \mathrm{RMB} / \mathrm{kWh}$ and $5 \mathrm{RMB} / \mathrm{kWh}$, respectively. The penalty price of aggregator $\mathrm{A}$ ranges from 5 to $30 \mathrm{RMB} / \mathrm{kWh}$. The simulation results show the total scheduling cost and payment cost of aggregator A, as presented in Figure 9.

From Figure 9, it can be observed that the payment cost and payment cost of aggregator A decrease with the increase 
TABLE 2: The parameters of IACs aggregators.

\begin{tabular}{|c|c|c|c|}
\hline & Aggregator A & Aggregator B & Aggregator C \\
\hline Minimum operating frequency $(\mathrm{Hz})$ & & 20 & \\
\hline Upper limit of user comfortable temperature $\left({ }^{\circ} \mathrm{C}\right)$ & & 28 & \\
\hline Setting temperature $\left({ }^{\circ} \mathrm{C}\right)$ & 23 & 24 & 25 \\
\hline Numbers of IACs & 100 & 300 & 200 \\
\hline Payment prices $(\mathrm{RMB} / \mathrm{kW})$ & 2 & 3 & 4 \\
\hline Punishment prices $(\mathrm{RMB} / \mathrm{kW})$ & 10 & 15 & 5 \\
\hline Confidence values of responsive stability constraint & & 0.9 & \\
\hline
\end{tabular}

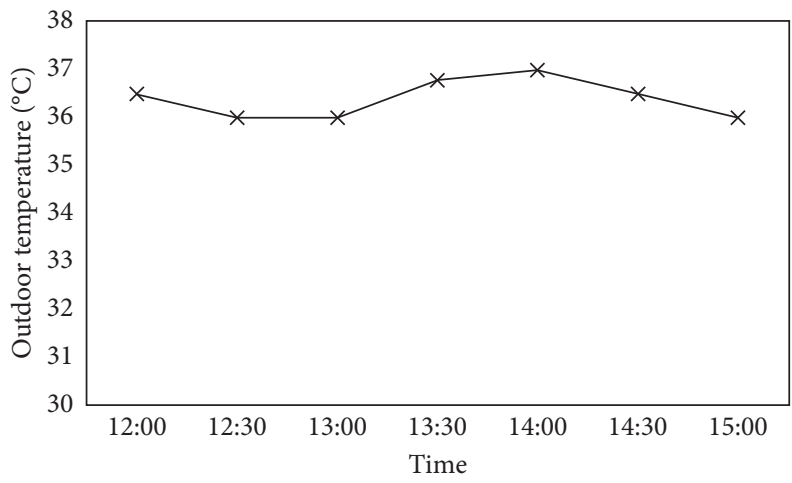

FIgURE 4: Outdoor temperature during power supply gap.

TABLE 3: The potential of IACs aggregators.

\begin{tabular}{|c|c|c|c|c|}
\hline \multicolumn{2}{|l|}{ Time } & Aggregator A & Aggregator B & Aggregator $\mathrm{C}$ \\
\hline \multirow{3}{*}{$\begin{array}{l}12: 00 \\
14: 30\end{array}$} & The potential of load reduction $(\mathrm{kW})$ & 41 & 90 & \multirow{3}{*}{$\begin{array}{c}40 \\
37.4\end{array}$} \\
\hline & Controllable duration (min) & 42.8 & \multirow{2}{*}{40.3} & \\
\hline & Total load reduction $(\mathrm{kW})$ & 171 & & \\
\hline \multirow{3}{*}{$\begin{array}{l}12: 30 \\
13: 00 \\
15: 00\end{array}$} & The potential of load reduction $(\mathrm{kW})$ & 35 & \multirow{3}{*}{$\begin{array}{c}75 \\
43.5\end{array}$} & \multirow{3}{*}{$\begin{array}{c}28 \\
38.7\end{array}$} \\
\hline & Controllable duration (min) & 48.6 & & \\
\hline & Total load reduction $(\mathrm{kW})$ & 138 & & \\
\hline \multirow{3}{*}{$13: 30$} & The potential of load reduction $(\mathrm{kW})$ & 44 & 99 & 44 \\
\hline & Controllable duration (min) & 41.5 & \multirow[t]{2}{*}{39} & \multirow[t]{2}{*}{33} \\
\hline & Total load reduction $(\mathrm{kW})$ & 187 & & \\
\hline \multirow{3}{*}{ 14:00 } & Potential of load reduction $(\mathrm{kW})$ & 46 & 105 & \multirow{3}{*}{30.2} \\
\hline & Controllable duration (min) & 38.6 & \multirow[t]{2}{*}{34.2} & \\
\hline & Total load reduction $(\mathrm{kW})$ & 201 & & \\
\hline \multirow{2}{*}{\multicolumn{2}{|c|}{$\begin{array}{l}\text { Variance of responsive deviation of single IAC } \\
\text { Variance of responsive deviation of IACs aggregators }\end{array}$}} & 0.15 & 0.06 & 0.14 \\
\hline & & 1.5 & 1.0 & 2.0 \\
\hline
\end{tabular}

of user penalty price. With the increase of penalty price, the scheduler gradually reduces the load reduction of aggregator A for optimizing cost, so the payment cost and payment cost of aggregator A are reduced.

5.5.3. Confidence of Responsive Stability. The confidence of responsive stability reflects the constraint on responsive deviation of aggregators. The larger the confidence value is, the more strict the constraint on responsive deviation of aggregators is. Suppose that the confidence values of aggregators $\mathrm{B}$ and $\mathrm{C}$ are all set to 0.9 and the confidence values of aggregator A vary from 0.9 to 0.95 . The simulation results show the payment cost and payment cost of aggregator A under different response-stability confidence, as presented in Figure 10.

It can be seen from Figure 10 that, with the increase of the confidence value of the responsive stability of aggregator $\mathrm{A}$, the response of aggregators becomes more stable, the risk of aggregator A is smaller, the uncertain-risk cost is reduced, and the payment cost is thus reduced. At the same time, the penalty cost of aggregator A decreases, and the payment cost of aggregator $\mathrm{A}$ increases. 


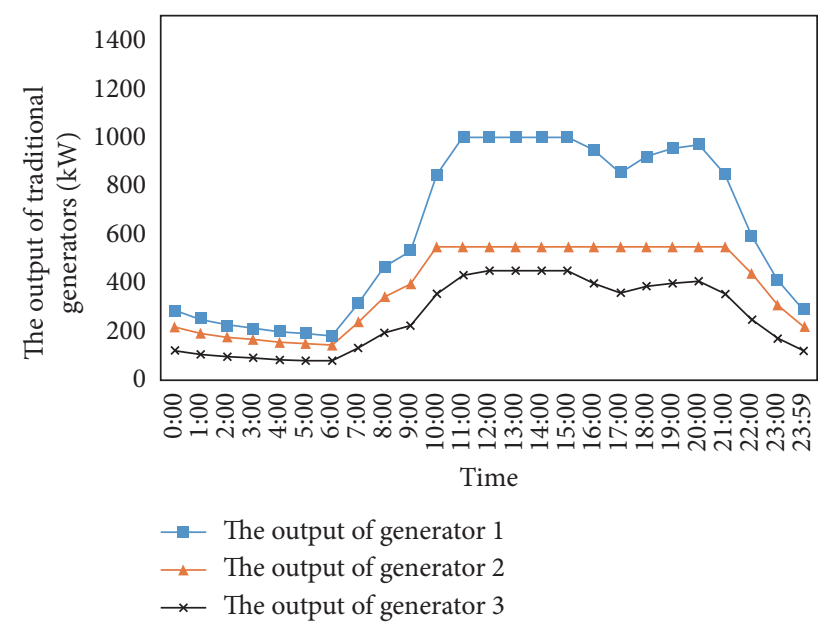

Figure 5: The output of the traditional generators.

Due to the influence of payment price, penalty price, and response-stability constraint, the payment cost and payment cost of aggregator A decrease with the increase of penalty price and the decrease of penalty price, while with the increase of response-stability confidence, the payment cost decreases and the payment cost increases.

Based on the generation-load coordinated scheduling optimization model, the grid scheduling department is more inclined to call the air-conditioning users with low payment price, high penalty price, and more reliable demand response for their own interests.

For IACs aggregators, when the payment price and penalty price are bound by the contract between schedulers and schedulable load, improving the reliability and stability of its demand response can increase the revenue of the aggregators. Therefore, according to the scheduling model in this paper, schedulers can reduce the scheduling cost as well as motivating air-conditioning users to improve their responsive stability, so as to improve the economy and reliability of power grid scheduling operation.

5.5.4. Risk Aversion Factors. The risk aversion factor $\lambda$ in the objective function can represent the degree of the concern of scheduler to the risk caused by the demand-response uncertainty of air-conditioning aggregators. With (20) as the objective function, the payment price, and responsedeviation variance of each IACs aggregator in Table 6 , as well as risk aversion factor ranging from 0 to 1 , we can derive the payment cost of air-conditioning aggregators through simulation, as shown in Figure 10. Obviously, from Figure 11, when the risk aversion factor is set to 0.6 , the payment cost is the smallest.

The column in Figure 11 represents the load reduction of each IACs aggregators during the power supply gap period from 13:30 to 14:00. We can observe the following:

(1) When $\lambda=0$, in order to minimize the total scheduling cost, the scheduler will call up aggregator $\mathrm{A}$ and then aggregator $\mathrm{B}$ according to the order of small to

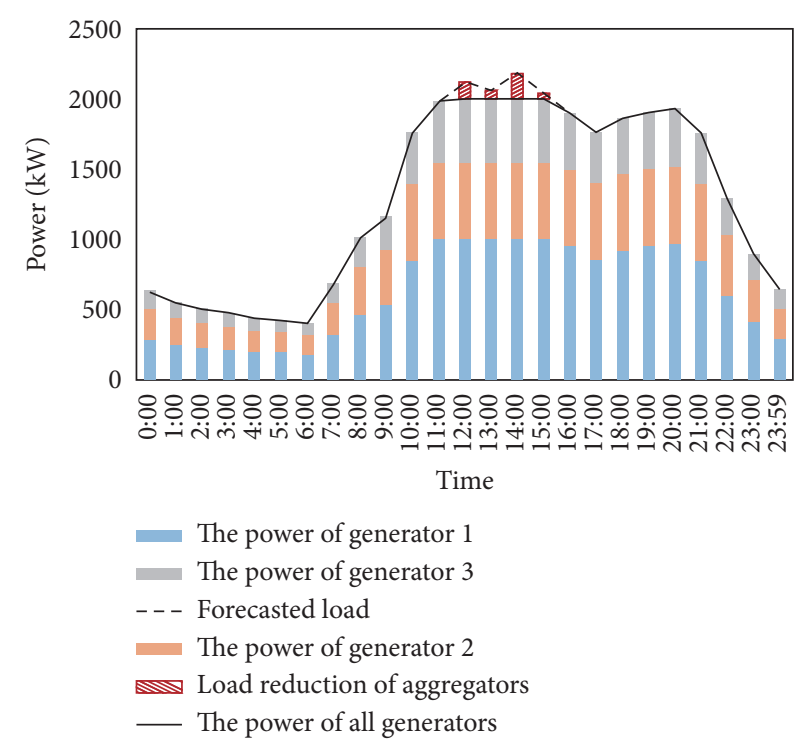

FIgURE 6: The output of the traditional generators and IACs aggregators.

TABle 4: Total load reduction of aggregators at each time.

\begin{tabular}{lc}
\hline Time & Load reduction $(\mathrm{kW})$ \\
\hline $12: 00$ & 115 \\
$12: 30$ & 88 \\
$13: 00$ & 59 \\
$13: 30$ & 120 \\
$14: 00$ & 178 \\
$14: 30$ & 108 \\
$15: 00$ & 41 \\
\hline
\end{tabular}

large payment price. However, the responsive reliability of aggregator A and aggregator B is poor, and the uncertain-risk cost is high, so the payment cost is high in this case.

(2) When $\lambda$ ranges from 0 to 0.5 , with the increase of $\lambda$, the scheduler will gradually pay more attention to the uncertain-risk cost of IACs aggregators. Therefore, it will gradually increase the call of IACs aggregators with smaller expected responsive deviation, then the uncertain cost will reduce, and hence the payment cost will gradually decrease.

(3) When $\lambda=0.6$, the scheduler will preferentially call aggregator $B$ with lower payment price and more reliable demand response, and the payment cost will also reach the minimum at this time.

(4) When $\lambda$ ranges from 0.6 to 1 , the scheduler pays more attention to the risk than the payment price. Meanwhile, with the increase of $\lambda$, more and more prefer to call the air-conditioning aggregators with higher responsive reliability. However, due to the fact that the payment price of the aggregators with higher responsive reliability is higher, the payment cost is on the rise with the increase of $\lambda$. 


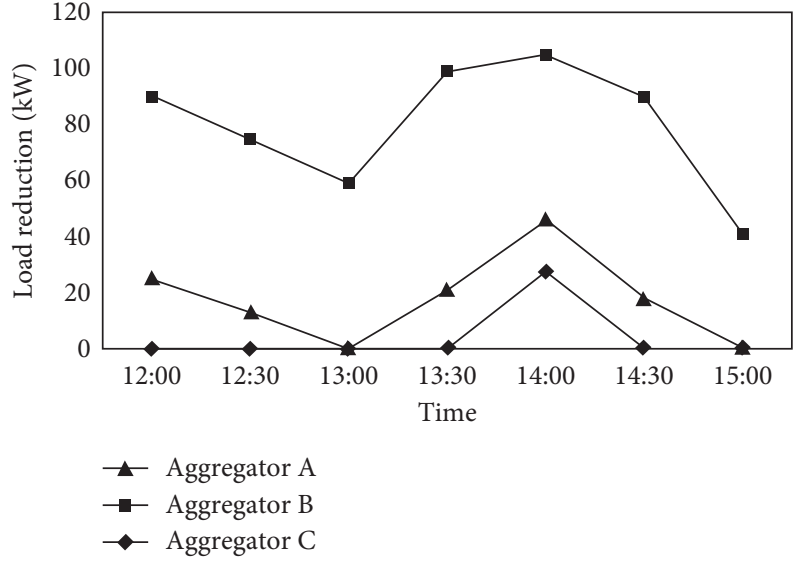

(a)

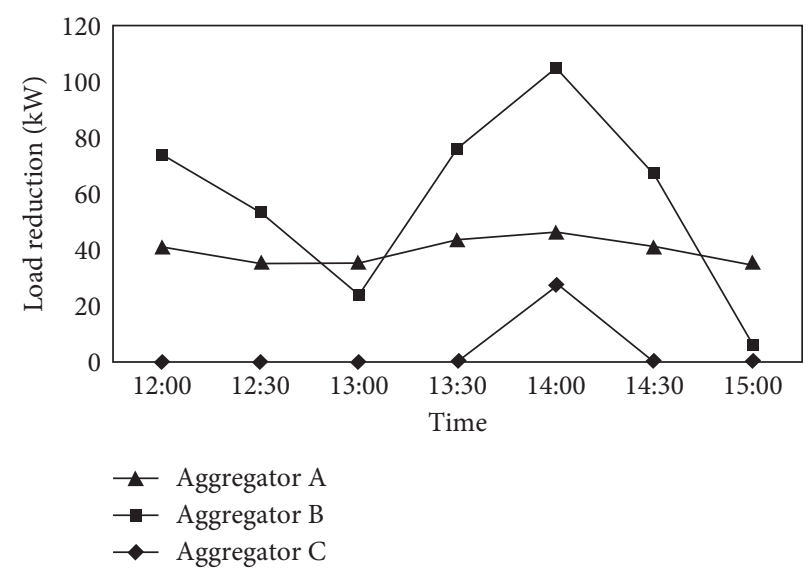

(b)

FIgURE 7: Load-reduction distribution of aggregators in (a) Case 1 and (b) Case 2.

TABLE 5: The cost of aggregators in both cases.

\begin{tabular}{lcc}
\hline Payment cost $(\mathrm{RMB})$ & $\begin{array}{c}\text { Compensation cost } \\
(\mathrm{RMB})\end{array}$ & $\begin{array}{c}\text { Payment cost } \\
(\mathrm{RMB})\end{array}$ \\
\hline Case 1 & 1031 & 1062.94 \\
1132.4 & & \\
\hline Case 2 & 1018 & 1162.22 \\
1275.7 &
\end{tabular}

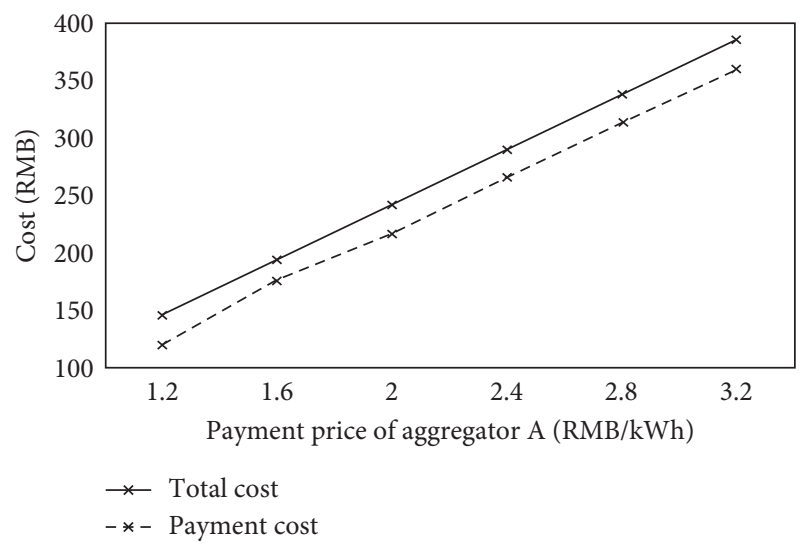

FIGURE 8: The payment cost and payment cost of aggregator A under different payment price.

(5) When $\lambda=1$, in order to avoid the risk, the scheduler will call air-conditioning aggregators according to the order of responsive reliability. However, the aggregators with better reliability have higher payment cost; hence the payment cost is not the minimum.

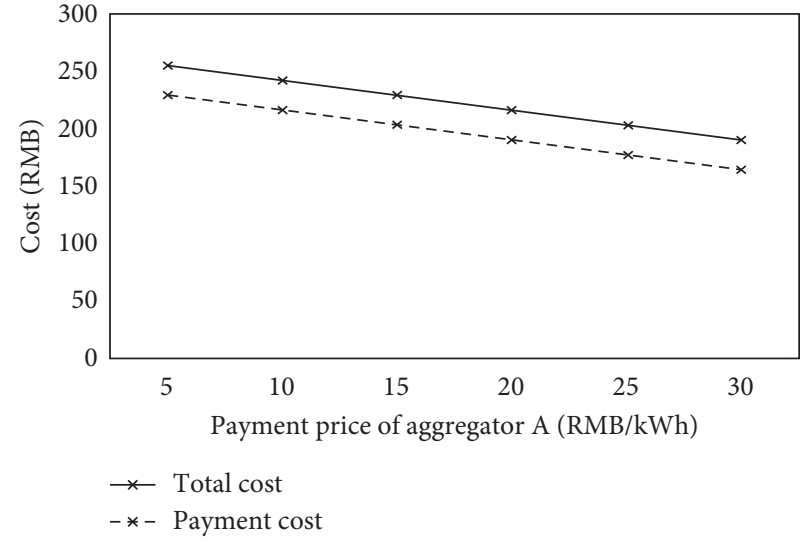

FIGURE 9: The payment cost and payment cost of aggregator A under different penalty price.

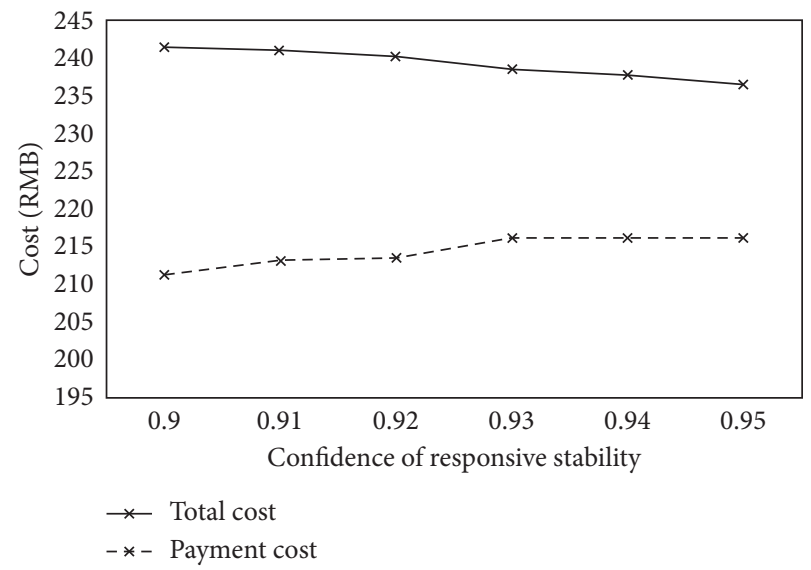

FIgURE 10: The payment cost and payment cost of aggregator A under different confidence of responsive stability. 
TABLE 6: The numerical setting of payment price and variance of responsive deviation.

\begin{tabular}{lccc}
\hline & Aggregator A & Aggregator B & Aggregator C \\
\hline Payment price (RMB/kwh) & 2 & 3 & 4 \\
Variance of responsive deviation & 2.0 & 1.5 & 1.0 \\
\hline
\end{tabular}

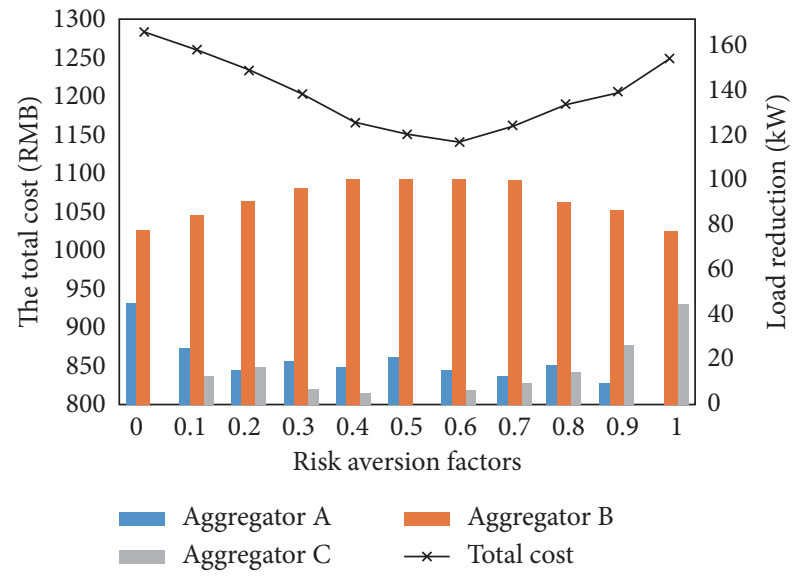

FIGURE 11: The payment cost and load reduction under different risk aversion factors.

\section{Conclusions}

Based on the evaluation of uncertain-risk cost and opportunity constraints, this paper proposes a day-ahead generation-load coordinated scheduling optimization model. According to the load forecasting, traditional generating units and IACs aggregators are coordinated to meet the power supply and demand balance in the period of insufficient power supply. Through this study, the following conclusions are derived:

(1) Based on the generation-load coordinated scheduling optimization, the scheduler is more inclined to call those IACs aggregators with low payment price, high penalty price, and more reliable demand response for their own interests. For IACs aggregators, under the condition that the payment price and penalty price are bound by the contract, improving the reliability and stability of demand response can increase the operational economy.

(2) The proposed coordinated scheduling optimization can reduce the scheduling cost of the power grid as well as motivating air-conditioning users to improve their responsive stability, so as to improve the economy and reliability of system operation.

(3) The risk aversion factor is very important to the economic operation of the system. Too large or too small risk factors are not conducive to optimizing the economic operation of the system.

\section{Data Availability}

The data used to support the findings of this study are included within the article.

\section{Conflicts of Interest}

The authors declare that there are no conflicts of interest regarding the publication of this paper.

\section{Acknowledgments}

This project was partly funded by the National Science Fundation of China (51577029) and Research Project of Jiangsu Power Company.

\section{References}

[1] N. K. Dhaliwal, F. Bouffard, and M. O’Malley, “A fast flexibility-driven generation portfolio planning method for sustainable power systems," IEEE Transactions on Sustainable Energy, (Early Access), 2020.

[2] M. C. Carreton and M. Carrion, "Generation capacity expansion considering reserve provision by wind power units," IEEE Transactions on Power Systems, (Early Access), 2020.

[3] C. Gu, W. Yang, Y. Song, and F. Li, "Distribution network pricing for uncertain load growth using fuzzy set theory," IEEE Transactions on Smart Grid, vol. 7, no. 4, pp. 1932-1940, 2016.

[4] S. Pineda and J. M. Morales, "Chronological time-period clustering for optimal capacity expansion planning with storage," IEEE Transactions on Power Systems, vol. 33, no. 6, pp. 7162-7170, 2018.

[5] C. Li, J. Shang, S. Zhu, L. Du, C. Wu, and Q. Wang, "An analysis of energy consumption caused by air temperature-affected accumulative effect of the air conditioning load," Automation of Electric Power System, vol. 34, no. 20, pp. 30-33, 2010.

[6] N. Mahdavi and J. H. Braslavsky, "Modelling and control of ensembles of variable-speed air conditioning loads for demand response," IEEE Transactions on Smart Grid (Early Access), 2020.

[7] K. Meng, Z. Y. Dong, Z. Xu, Y. Zheng, and D. J. Hill, "Coordinated dispatch of virtual energy storage systems in smart distribution networks for loading management," IEEE Transactions on Systems, Man, and Cybernetics: Systems, vol. 49, no. 4, pp. 776-786, 2019.

[8] X. Hu and J. Nutaro, "A priority-based control strategy and performance bound for aggregated HVAC-based load shaping," IEEE Transactions on Smart Grid (Early Access), 2020.

[9] W. Cui, Y. Ding, H. Hui et al., "Evaluation and sequential dispatch of operating reserve provided by air conditioners considering lead-lag rebound effect," IEEE Transactions on Power Systems, vol. 33, no. 6, pp. 6935-6950, Nov. 2018.

[10] X. Gao, K. Meng, D. Wang, G. Liang, F. Luo, and Z. Y. Dong, "Optimal wind turbine and air conditioner loads control in distribution networks through MILP approach," in Proceedings of 2016 IEEE Power and Energy Society General Meeting, IEEE, Boston, MA, USA, pp. 1-5, July 2016.

[11] T. Remani, E. A. Jasmin, and T. P. I. Ahamed, "Residential load scheduling with renewable generation in the smart grid: a reinforcement learning approach," IEEE Systems Journal, vol. 13, no. 3, pp. 3283-3294, 2019.

[12] B. Sohet, Y. Hayel, O. Beaude, and A. Jeandin, "Coupled charging-and-driving incentives design for electric vehicles in 
urban networks," IEEE Transactions on Intelligent Transportation Systems (Early Access), 2020.

[13] H. Khani and H. E. Z. Farag, "Optimal day-ahead scheduling of power-to-gas energy storage and gas load management in wholesale electricity and gas markets," IEEE Transactions on Sustainable Energy, vol. 9, no. 2, pp. 940-951, 2018.

[14] T. Lee, M. Cho, Y. Hsiao, P. Chao, and F. Fang, "Optimization and implementation of a load control scheduler using relaxed dynamic programming for large air conditioner loads," IEEE Transactions on Power Systems, vol. 23, no. 2, pp. 691-702, 2008.

[15] F. Luo, J. Zhao, Z. Y. Dong et al., "Optimal dispatch of air conditioner loads in southern China region by direct load control," IEEE Transactions on Smart Grid, vol. 7, no. 1, pp. 439-450, 2016.

[16] N. Chakraborty, A. Mondal, and S. Mondal, "Intelligent scheduling of thermostatic devices for efficient energy management in smart grid," IEEE Transactions on Industrial Informatics, vol. 13, no. 6, pp. 2899-2910, Dec. 2017.

[17] H. Hui, Y. Ding, T. Chen, S. Rahman, and Y. Song, "Dynamic and stability analysis of the power system with the control loop of inverter air conditioners," IEEE Transactions on Industrial Electronics (Early Access), 2020.

[18] M. Hu and F. Xiao, "Price-responsive model-based optimal demand response control of inverter air conditioners using genetic algorithm," Applied Energy, vol. 219, pp. 151-164, 2018.

[19] Y. Che, J. Yang, Y. Zhou, Y. Zhao, W. He, and J. Wu, "Demand response from the control of aggregated inverter air conditioners," IEEE Access, vol. 7, pp. 88163-88173, 2019.

[20] B. Wang, T. Zhang, X. Hu, Y. Bao, and H. Su, "Consensus control strategy of an inverter air conditioning group for renewable energy integration based on the demand response," IET Renewable Power Generation, vol. 12, no. 14, pp. 16331639, 2018.

[21] H. Hui, Y. Ding, Z. Lin, P. Siano, and Y. Song, "Capacity allocation and optimal control of inverter air conditioners considering area control error in multi-area power systems," IEEE Transactions on Power Systems, vol. 35, no. 1, pp. 332$345,2020$.

[22] H. Hui, Y. Ding, and M. Zheng, "Equivalent modeling of inverter air conditioners for providing frequency regulation service," IEEE Transactions on Industrial Electronics, vol. 66, no. 2, pp. 1413-1423, 2019.

[23] X. Zhuang, C. Ye, Y. Ding, H. Hui, and B. Zou, "Data-driven reserve allocation with frequency security constraint considering inverter air conditioners," IEEE Access, vol. 7, pp. 120014120022, 2019. 